\title{
Photophysical and photochemical applications of femtosecond time-resolved transient absorption spectroscopy
}

\author{
Paolo Foggi, ${ }^{\dagger}$ Laura Bussotti, and Frederik V. R. Neuwahl \\ LENS and Istituto Nazionale per la Fisica della Materia Largo E. Fermi 2 - 50125 Firenze, Italy
}

\begin{abstract}
The aim of this paper is to provide the main pieces of information concerning the application of transient absorption (TA) spectroscopy with sub-picosecond laser pulses. A description of the experimental apparatus and of some detection schemes are included together with the most common mathematical formulas utilized to analyze the signals. The results, recently obtained in our laboratory and presented here, concern the investigation of the excited state dynamics of simple molecular systems. Examples of the measurements of the relaxation processes occurring in the lowest excited states of some aromatic molecules will be discussed in order to show the potentiality of the technique.
\end{abstract}

\section{INTRODUCTION}

In the past decade a tremendous expansion of the research activity in the field of femtosecond laser spectroscopies occurred [1-6]. Many research groups around the world operating in different fields from physics and chemistry to biology, medicine and material science, utilize laser setups capable of providing subpicosecond pulses. Femtochemistry is one of the revolutionary ideas in the field of chemistry and has been recently awarded with the Nobel prize to A. H. Zewail [7].

Femtosecond transient absorption (TA) spectroscopy represents the natural evolution of laser flash photolysis since the introduction of ultrashort laser sources [8]. The principles ruling the technique are essentially the same though much more care is necessary if one wants to utilize at the best the resolution given by the laser system [9]. Therefore a description of the setup and of the sample handling is essential in understanding the experimental details which allows to fully exploit the capability of this technique.

With pulse duration of about $100 \mathrm{fs}$ it is nowadays possible to access most of the dynamics occurring in the excited electronic states: internal conversion, intramolecular vibrational relaxation and many other processes leading to reactive pathways. The technique is somehow complementary to time-resolved fluorescence, though in principle it has a larger content of information. In fact, while time-resolved fluorescence measurements are attainable only with reasonably fluorescent samples (with a quantum yield of $\sim 1 \%$ ), TA can be utilized in any system. The only requirement is

\footnotetext{
† Department of Chemistry, University of Perugia, via Elce di Sotto 8 - 06100 Perugia, Italy

E-mail: foggi@lens.unifi.it
}

that a transition from an excited state to higher excited states is allowed and possesses a dipole moment strong enough to make it observable. This is highly probable if the observation is extended in a wide spectral range (from mid IR up to UV).

This paper discusses some of the advantageous features of TA spectroscopy and provides examples to illustrate them. It is organized in four sections including the present introduction. A description of the experimental setup follows. A third section is devoted to the analysis of the signals. The last section regards the applications of TA in the study of intramolecular relaxation processes.

\section{THE FEMTOSECOND LASER APPARATUS}

Most of the femtosecond systems working in research laboratories around the world are nowadays based on Ti:sapphire. The Ti:sapphire system utilized for the experiments described in the present article is schematically represented in Figure 1. As it has been extensively described in previous papers [9-11], only a brief description is given here.

The master oscillator is a Ti:sapphire laser excited by a cw diode pumped intracavity doubled Nd:YVO. The laser provides a train of femtosecond pulses ( $<70 \mathrm{fs})$ at $80 \mathrm{MHz}$ repetition rate with $0.5 \mathrm{~W}$ of average power at the central wavelength of $800 \mathrm{~nm}$. The energy/pulse is about $6 \mathrm{~nJ}$, while for many experiments in photophysics and photochemistry higher energy/pulse is needed. In addition, the system is only tunable from 750 to $850 \mathrm{~nm}$ and tunability can be achieved by non linear optical processes. In order to get higher energy/pulse the pulses are amplified. In the present case the amplification is obtained by injecting the pulses in a Ti:sapphire regenerative amplifier pumped at $1 \mathrm{kHz}$ by a Q-switched, in- 


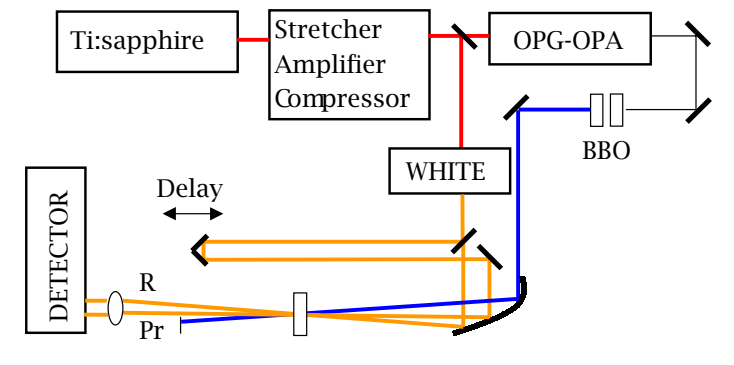

Figure 1. The femtosecond pump-probe spectrometer. The detector can be a CCD camera coupled to a flat field monochromator or a double photodiode and an interference filter which selects a portion of the white pulse spectrum.

tracavity doubled Nd:YLF. Stretching of the pulse duration and the following compression after amplification are needed in order to prevent damage of the laser medium during the amplification process. The output after the compression stage is $700 \mathrm{~mW}$ at $1 \mathrm{kHz}$ and the pulse duration is $90 \mathrm{fs}$.

Frequency mixing and parametric processes in BBO crystals are utilized to extend the tunability of the system [9].

For pump-probe experiments two independently tunable and possibly synchronized pulses are needed. This latter requirement is achieved by the split-beam technique. The amplified pulse at $800 \mathrm{~nm}$ is divided into two parts whose relative intensity is chosen depending on the experiment. The two pulses travel through different optical paths and their relative delay is controlled by an optical delay line with micrometric precision ( $30 \mu \mathrm{m} \Rightarrow 100 \mathrm{fs}$ ). One part is generally utilize to pump an optical parametric generator and amplifier (OPG-OPA) [12,13] or to produce the second or the third harmonic of the fundamental.

Among the processes utilized for the production of the probe pulse the most flexible is white continuum generation [9]. The white pulse is obtained by tightly focussing $1-2 \mathrm{~mJ} /$ pulse of fundamental radiation at $800 \mathrm{~nm}$ into a thin $\mathrm{CaF}_{2}$ plate. The spectrum is broad and contains sufficiently intense components from $250 \mathrm{~nm}$ in the UV to $1.4 \mu \mathrm{m}$ in the NIR. Multichannel detection for transient spectroscopy is achieved by sending the white continuum after passing through the sample to a flat field monochromator coupled to a CCD detector. Alternatively the transmitted intensity of a portion of light components selected by an interference filter is detected by a photodiode as a function of the delay in order to measure the rise and the decay of a particular process. To avoid the observation of relaxation phenomena not directly connected to excited state dynamics, measurements are made at the so called "magic angle": excitation and probe are linearly polarized at $54.7^{\circ}$ one respect to the other [14].

\section{DATA ANALYSIS}

3.1. Spectral data. As in the case of classical absorption spectroscopy, two beams are generally required to measure the variation of transmittance in the excited volume. The beams must have the same spectral distribution and therefore they must derive from the same source (in the present case the probe pulse). One pulse is spatially and temporally overlapped to the excitation pulse and has the function of the probe. The other passes through the sample in a different position or it is spatially overlapped but temporally anticipated in respect to the excitation pulse and has the function of the reference beam.

The transient transmittance $T(\tau, \lambda)$ at a given wavelength $\lambda$ is defined as the ratio $I(\tau, \lambda) / I_{0}(\lambda)$, where $I$ and $I_{0}$ are the probe intensity measured respectively with and without the pump pulse. Therefore $T(\tau, \lambda)$ is obtained by two subsequent acquisitions. In order to reduce the effect of the fluctuation of spectral distribution the intensity of the probe is divided by the intensity $I_{r}$ of the reference.

$$
T(\tau, \lambda)=\frac{I(\tau, \lambda)}{I(\lambda)_{r}} \cdot \frac{I(\lambda)_{r}}{I(\lambda)_{0}} .
$$

$I_{r}$ never depends on the presence of the pump and vanishes in the division giving rise to $T(\tau, \lambda)$. The total light intensity seen by the detector is not only due to $I(\tau, \lambda)$, $I_{r}(\lambda)$ and $I_{0}(\lambda)$ but it is also due to the scattered light from the pump and, when present, to the fluorescence of the excited sample. These latter two contributions can be minimized by the insertion of pinholes in the optical path of both probe and reference beams after the cell. Nevertheless they can still be significant in comparison to the variation of intensity of the probe due to the absorption process. It can be therefore necessary to subtract the scattered light contribution from the overall signal. Let $F_{p}$ and $F_{r}$ indicate the amount of spurious light seen by the detector superimposed to the probe and reference beam respectively, i.e. all the fluorescence and scattered light that depends on the pump. Three consecutive acquisitions are made in order to measure:

1) $I+F_{p}, I_{r}+F_{r}$ with both pump and probe passing through the sample;

2) $I_{0}, I_{r}$ with only the probe;

3) $F_{p}, F_{r}$ with only the pump.

$I$ and $I_{0}$ are then obtained by subtracting the intensities read while the probe is blocked from those read while both the pump and the probe are on

$$
T(\tau, \lambda)=\frac{\left[I(\tau, \lambda)+F_{p}\right]-F_{p}}{\left[I_{r}(\lambda)+F_{r}\right]-F_{r}} \cdot \frac{I(\lambda)_{r}}{I(\lambda)_{0}} .
$$



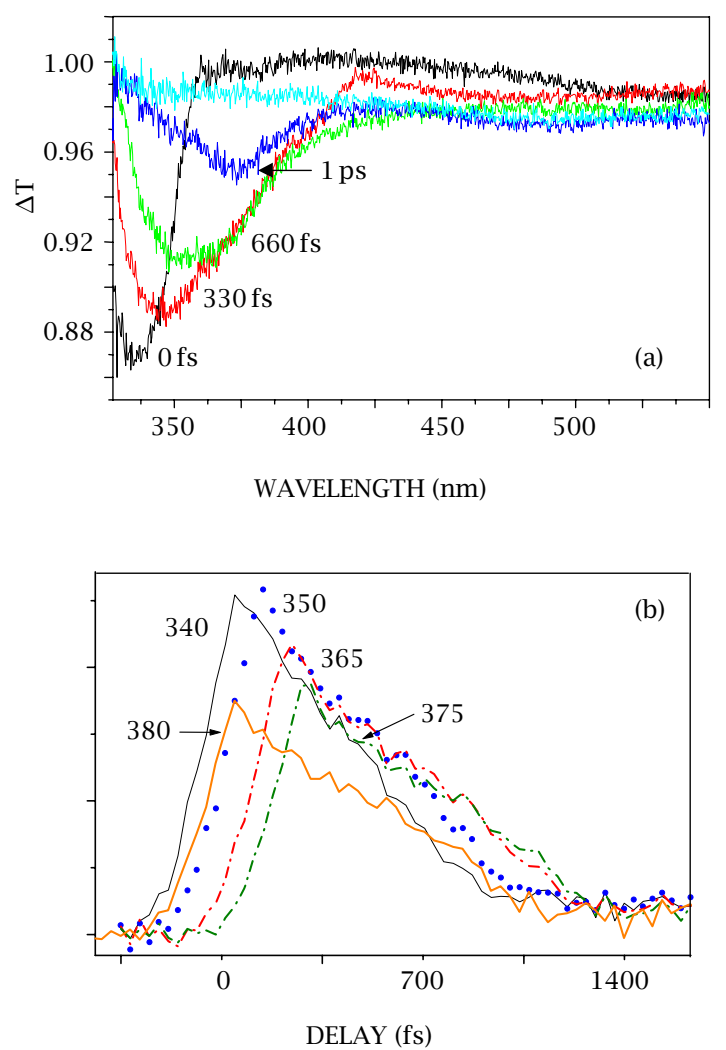

Figure 2. (a) Spectral evolution of the TA spectrum of $\mathrm{CH}_{2} \mathrm{Cl}_{2}$ due to dispersion. (b) The temporal dispersion as a function of wavelength: the maximum of each curve is shifted to positive delay values for increasing wavelengths.

This is the general formula utilized to plot the transient spectra.

3.2. The white dispersion correction. When white continuum probe is utilized, one has to face a problem occurring in the first picosecond after excitation. White continuum is temporally dispersed when it crosses any optical component such as beam splitters, lenses, cells. In our setup all the optical components are reflective. However the pulse has to travel through the $\mathrm{CaF}_{2}$ plate where it is generated and to cross the inlet window of the sample cell. Modeling of the dispersion introduced by the crossed optics has been already discussed [9]. A dispersed pulse has longer wavelength components in its front and shorter wavelengths in its tail (positive chirping). As a result, a sample absorbing in a wide spectral region shows a spectrum with absorption bands in the blue at shorter delays and in the red at longer ones. This evolution has nothing to do with any of the molecular processes occurring after excitation. The artifact is due to the probing process which occur at negative delays for the blue components and at positive delays for the red. One way to solve the problem is to optically compensate

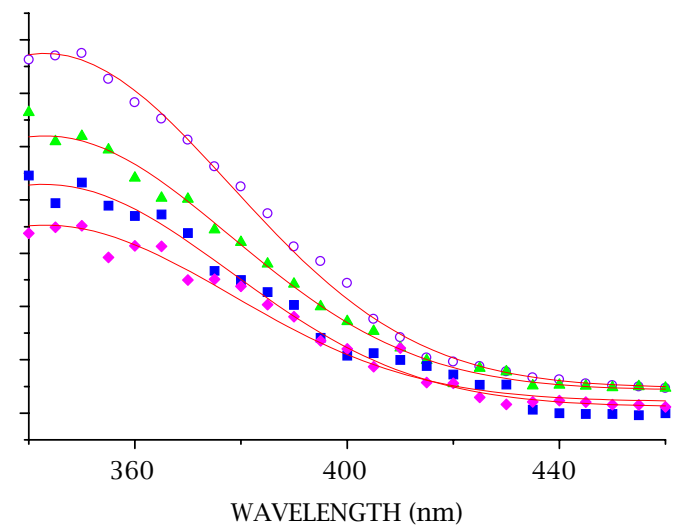

Figure 3. The TA spectra of $\mathrm{CH}_{2} \mathrm{Cl}_{2}$ after the dispersion compensation at four different delays from 0 to $400 \mathrm{fs}$.

the dispersion. This solution is not straightforward, it introduces additional optics in the experimental set up and makes it less reliable. A second solution is to correct numerically the spectra. In order to understand the spectral evolution of the transient species it is necessary to characterize the dispersion of white pulse. Once this latter is well defined it is always possible to reconstruct the spectra. (see Figures 2, 3).

Sometimes chirped pulses can be useful to disentangle different contribution to the signal (see below).

3.3. Kinetics. The TA is a third order non-linear process in which three fields, two from the pump and one from the probe, interact in the sample $[15,16]$. The third order polarization beats with a second field deriving from the probe to give rise to a modulated transmitted intensity. Because of the presence of a real state in resonance with the pump field, the molecular system is able to memorize, for a certain amount of time, the effects of the interaction and the polarization lasts beyond the time the pump pulse is switched off. Such situation is represented by the following integral.

$$
S(\tau)=\int_{-\infty}^{\infty} d t \int_{-\infty}^{\infty} d t^{\prime} I_{\text {probe }}(t-\tau) I_{\text {pump }}\left(t-t^{\prime}\right) R\left(t^{\prime}\right) .
$$

In equation (3) it is possible to distinguish two parts, the molecular response and the instrumental function. It can be rewritten as follows

$$
S(\tau)=\int_{-\infty}^{\infty} d t^{\prime} g\left(t^{\prime}-\tau\right) R\left(t^{\prime}\right)
$$

where $g(t)$ is the second order pump-probe crosscorrelation, i.e. the instrumental function.

$$
g\left(t^{\prime}-\tau\right)=\int_{-\infty}^{\infty} d t I_{\text {probe }}(t-\tau) I_{\text {pump }}\left(t-t^{\prime}\right) .
$$

Equation (4) is a correlation integral, but in the case of symmetrical pulse envelopes it can easily be trans- 
formed in a convolution integral. The zero delay is defined when the pump and the probe are totally overlapped. Cross-correlation is determined utilizing a material with an instantaneous response: $R(t)$ in equation (4) transforms into a Dirac's delta function. Typically a second order non resonant process is utilized, like sum or difference frequency mixing in a non linear crystal. This however implies some small experimental changes, in respect to the setup for TA measurements, which may affect the time resolution of the instrumentation. This aspect is important when very fast processes (in comparison to the instrumental function) are observed.

An alternative is to utilize the non-resonant response of the sample itself. If solutions are under investigation, a convenient way to determine the instrumental function directly in the sample is to observe the stimulated Raman gain of some vibrational modes of the solvent [9]. Corrections to the instrumental function determined according to the latter procedure must be introduced in order to take into account the effects of group velocity mismatch when pump and probe central wavelengths are far apart [9].

\section{APPLICATIONS TO THE STUDY OF INTRAMOLECULAR PROCESSES}

Rigorously speaking, only those processes occurring in isolated molecules can be defined intramolecular processes. When solutions are considered, as in the present case, it must be taken into account the interaction with the surrounding solvent molecules which somehow modulate the dynamical processes. However, very often the solvent provides only a heat sink: the energy exchange between solute and solvent occurs at very low frequencies and therefore the dynamics has a larger time constant than that of primary events. Therefore all the experiments described in the present section are discussed as if they occurred in isolated molecules. A comprehensive picture of all possible intramolecular processes is given by the Jablonski diagram. Following that scheme it is possible to give a qualitative sequence of events which has a general validity [17].

The interaction of visible or UV light with a molecular system generally produces a vibrationally hot electronic state. The primary event following excitation is therefore vibrational relaxation. The way it occurs depends on the vibrational modes involved, on the coupling between them and on the shape of the multidimensional potential surface of the excited state. Afterwards the excited state may exchange energy to vibrations of a lower state with the same multiplicity (internal conversion) or different multiplicity (intersystem crossing). In addition, reactive channels can exist which speed up the decay of the excited state.

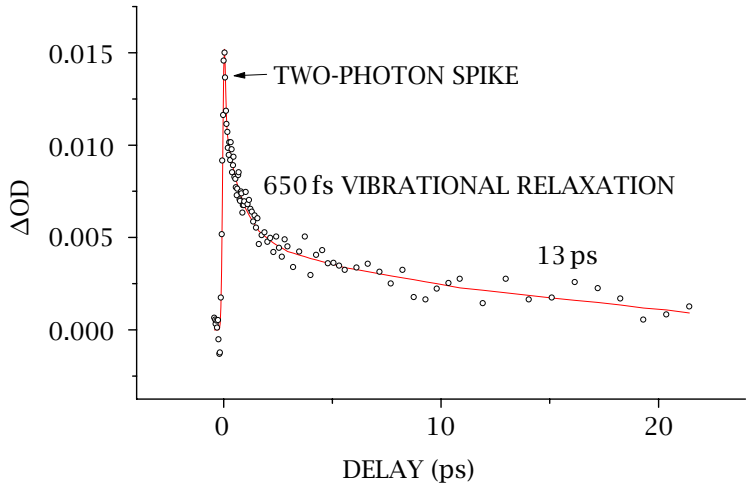

Figure 4. The two-photon contribution and the fast vibrational component of the decay of $\mathrm{S}_{3}$ excited electronic state of 1,3,5-tri-t-butyl-pentalene [19].

4.1. Coherent processes. At very short times, dephasing processes rapidly destroy the coherence introduced by light matter interaction. When the pulses are longer in comparison to dephasing time the effects are averaged out and only incoherent processes are observed. Pulses with duration comparable to or shorter than coherence time allow the observation of other effects. For example, it is possible to observe often a spike on top of a decay curve [18]. The maximum of the spike correspond to zero delay time. Such effect can be reproduced numerically by introducing in the response function $R(t)$ of equation (4) a term with fast rise and decay, in practice a delta function (see Figure 4). The simplest interpretation of this sharp signal is that it is due to two-photon absorption occurring only when pump and probe are temporally overlapped in the sample.

In some cases chirped pulses can help to distinguish between coherent and incoherent processes. In Figure 5 the interference between two-photon (coher-

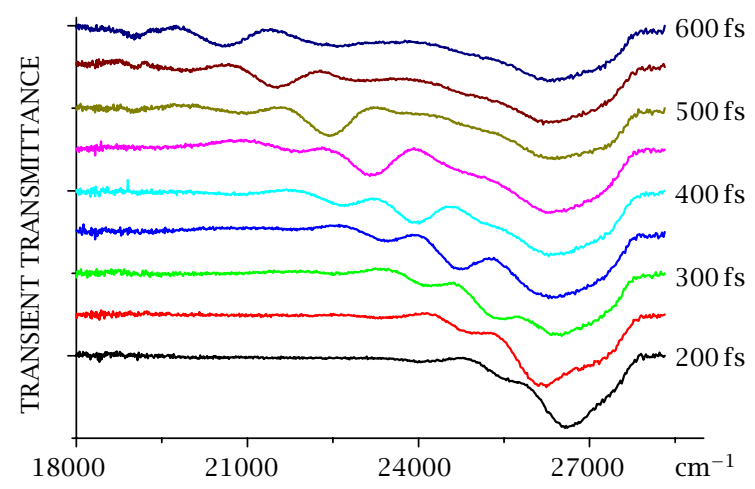

Figure 5. The effect of white dispersion and coherent/population interference in the TA spectrum of $\mathrm{S}_{1}$ excited electronic state of azulene in methanol. 
ent) absorption and transient (population) absorption is clearly apparent. The intensity modulation is observed only when the excitation pulse and one component of the white are temporally overlapped. It is a real interference due to cross terms between the population and the coherence contributions to the overall signal. Because the white pulse is dispersed the modulation moves as a function of the delay. Such effect can't be observed in the frequency domain with unchirped pulses.

\subsection{Vibrational relaxation and internal conver-}

sion. After the two-photon spike a very fast decay is generally observed with time constants ranging from $100 \mathrm{fs}$ up to $1 \mathrm{ps}$, depending on the complexity of the molecular system (see Figure 4). This decay is generally associated to the vibrational relaxation. Such relaxation is due to the anharmonic coupling between vibrational modes.

Internal conversion (IC) is the transfer of energy from a thermalized excited electronic state to the vibrational manifold of a lower electronic state. IC can be as fast as vibrational relaxation when high energy levels are involved. For example, in the case of pyrene $S_{2} \rightarrow S_{1}$ transition occurs with a time constant of $\sim 100 \mathrm{fs}$ (see Figure 6) [20]. In some cases specific couplings with vibrational levels open preferential channels of the decay process (see Figure 7).

4.3. Intersystem crossing. Intersystem crossing (ISC) is forbidden by rules of conservation of angular momentum. As a consequence, ISC generally occurs on very long time scales. However the ElSayed's rule [17] states that organic molecules with lowest excited sin-

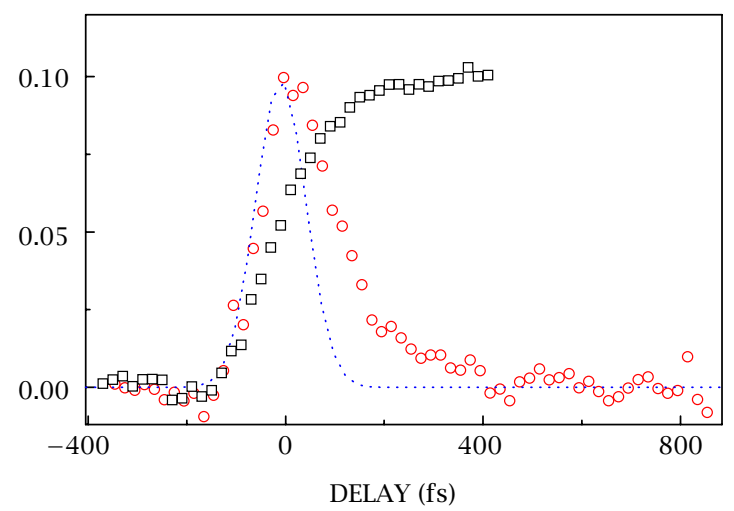

Figure 6. $\mathrm{S}_{2} \rightarrow \mathrm{S}_{1}$ internal conversion in pyrene. The signal at $640 \mathrm{~nm}$ (open circles) is due only to an $\mathrm{S}_{2} \rightarrow \mathrm{S}_{\mathrm{n}}$ transition, grows instantaneously and decays exponentially with a time constant of $100 \mathrm{fs}$. The signal at $480 \mathrm{~nm}$ (open squares) is due to an $\mathrm{S}_{1} \rightarrow \mathrm{S}_{\mathrm{m}}$ transition and grows with a time constant of $100 \mathrm{fs}$. The dotted line is the instrumental function of 120 fs FWHM.

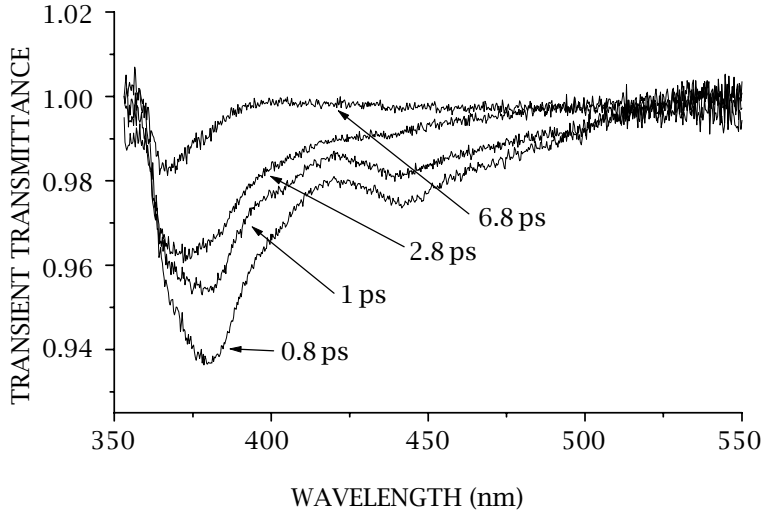

Figure 7. The $\mathrm{S}_{1} \rightarrow \mathrm{S}_{0}$ internal conversion of azulene in cyclohexane. The TA spectrum originating from $\mathrm{S}_{1}$ excited state disappears with an exponential law with a time constant of $1.2 \mathrm{ps}$. The band at $360 \mathrm{~nm}$ is due to an hot transition of the ground state. Its decay is exponential and has a time constant of $7 \mathrm{ps}$.

glet states of $n \pi^{*}$ configuration often exhibit very fast ISC. This is, for example, the case of the molecule of isophtalaldehyde.

The existence of two carbonilic groups in isophtalaldehyde helps the opening of non radiative electronic relaxation pathways by spin orbit coupling. As a matter of fact the TA technique allows to measure the rise of a strong absorption signal which lasts for microseconds. The absorption is due to a $\mathrm{T}_{1} \rightarrow \mathrm{T}_{\mathrm{n}}$ transition. The rise time is of the order of 8 ps (see Figure 8 ).

4.4. Intramolecular proton transfer. The final examples reported in the present article concerns one of the simplest photo-reactions: the excited state intramolecular proton transfer (ESIPT) $[11,21]$. The ex-

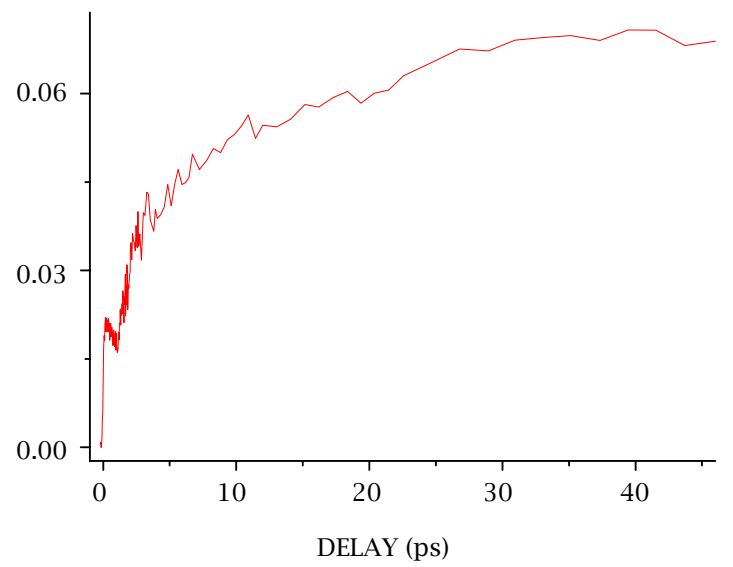

Figure 8. The fast growing ( 8 ps) of the triplet absorption in isophtalaldehyde $\left\{\left(\mathrm{C}_{6} \mathrm{H}_{4}\right)-1,3-(\mathrm{CHO})_{2}\right\}$. 
ample of the molecule of [2,2'-bipyridil]-3,3'-diol is discussed.

In this highly symmetric molecule the presence of two intramolecular hydrogen bonds open the possibility to two distinct mechanisms:

i) concerted transfer of two protons;

ii) two-step mechanism.

It has been shown that the dynamics depends on the probe wavelength and three different signals are identified [11]. The first grows fast and cannot be resolved with the present time resolution. It is possible only to put an upper limit of $50 \mathrm{fs}$ to the formation of the transient species. It is observed a second signal increasing with a time constant of about 10 ps. Finally, in a particular region of the TA spectrum a decay of about $20 \mathrm{ps}$ is also observed (see Figure 9). The model which accounts for the observed dynamical behavior can be summarized as it follows. After the excitation the $S_{1}$ state of the molecule has three possible decay channels:

1) the fast transfer of one or both the protons;

2) the fast formation a state which does not undergo ESIPT;

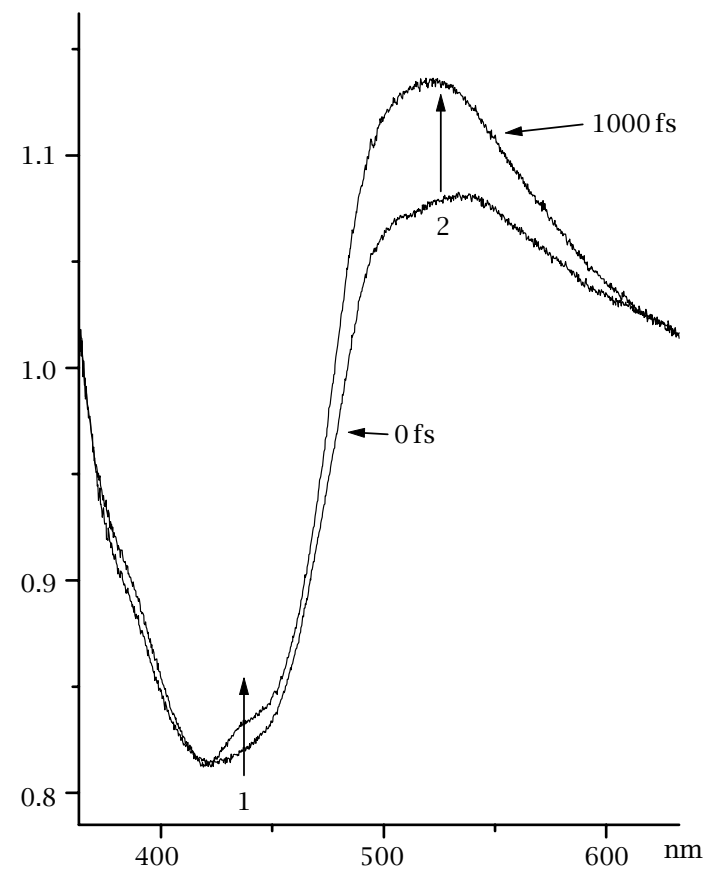

Figure 9. The TA spectrum of [2,2'-bipyridil]-3,3'-diol. After 1 ps the absorption (arrow 1) becomes more structured. The decay of the intensity in position 1 has a time constant of 20 ps. The stimulated emission (arrow 2) becomes more intense. This is interpreted as due to the transfer of the second proton. Intensity increases with a time constant of about 10 ps.
3) a slow transfer of the second proton.

Point 1 is confirmed by the instantaneous formation of a stimulated emission signal. Point 2 is consistent with the observation of a decay only in the TA intensity. Point 3 three is consistent with the fact that the intensity of the stimulated emission grows in the following 20-30 ps and becomes slower in polar solvents. The signal described in point 2 is due to a state with $n \pi^{*}$ character with a lifetime of the order of $20 \mathrm{ps}$ as in the case of the parent molecule dipyridine.

\section{CONCLUSIONS}

The examples reported in this article illustrate the capability of femtosecond absorption spectroscopy. The technique has reached a very high level of sensitivity and temporal resolution and can satisfy most of the requirements of researchers working in the field of physical and biophysical chemistry. 100 fs resolution is nowadays a feasible goal for many research groups in the field of time resolved spectroscopy and for many purposes it represent an adequate tool for the investigation of dynamical processes occurring in molecular systems of any complexity. Concluding with the words of George Porter, we have the impression that "chemists are near the end of the race against time" [8]. A higher time resolution, which however requires bigger efforts, is only needed when one wants to investigate more subtle effects of fundamental importance in the comprehension of the basic principles ruling light-matter interaction.

\section{ACKNOLEDGEMENTS}

This work has been supported by the Italian Ministero dell' Università e della Ricerca Scientifica e Tecnologica (MURST) and by the commission of the Europeam Communiteis under the contract \# HPRI-CT1999-00111. Authors wish to acknowledge the essential contribution of R. Ballerini and H. Hajeb of the machine shop and of M. De Pas and M. Giuntini of the electronic shop of LENS.

\section{References}

[1] Femtochemistry and Femtobiology, V. Sundström (ed.), World Scientific, Singapore, 1997.

[2] Femtochemistry, M. Chergui (ed.), World Scientific, Singapore, 1996.

[3] Femtosecond Chemistry, J. Manz and L. Wöste (eds.), Vol. 1 and 2, VCH, Weinheim, 1995.

[4] Femtosecond Reaction Dynamics, D. A. Wiersma (ed.), North Holland, Amsterdam, 1994.

[5] Ultrafast Processes in Chemistry and Biology Chemistry for the 21st Century, M. A. El-Sayed, I. Tanaka, and Y. N. Molin (eds.), IUPAC, Blackwell Scientific, Oxford, 1994. 
[6] E. Schreiber, Femtosecond Real-Time Spectroscopy of Small Molecules and Clusters, Springer, New York, 1998.

[7] A. H. Zewail, J. Phys. Chem. 104 (2000), 5693; and references therein.

[8] G. Porter, Femtosecond Chemistry, J. Manz and L. Wöste (eds.), Vol. 1, VCH Weinheim, 1995, p. 3.

[9] F. V. R. Neuwhal, L. Bussotti, and P. Foggi, Research Advances in Photochemistry and Photobiology, Vol. 1, Global Research Network, Trivandrum, Kerala, India, 2000, p. 77.

[10] L. Bussotti, B. Danieli, M. D’Auria, P. Foggi, G. Lesma, and R. Righini, Photochem. Photobiol. 71 (2000), 29.

[11] F. V. R. Neuwhal, P. Foggi, and R. Brown, Chem. Phys. Lett. 319 (2000), 157.

[12] I. M. Bayanov, R. Danielius, P. Heinz, and A. Seilmeier, Opt. Commun. 113 (1994), 99.

[13] R. Danielius, A. Piskarskas, P. Di Trapani, A. Andreoni, C. Solcia, and P. Foggi, Appl. Opt. 35
(1996), 5336.

[14] G. Fleming, Chemical Application of Ultrafast Spectroscopy, Oxford University Press, New York, 1986.

[15] W. T. Pollard and R. A. Mathies, Annu. Rev. Phys. Chem. 43 (1992), 497.

[16] S. Mukamel, Principles of Nonlinear Optical Spectroscopy, Oxford University Press, Oxford, 1995.

[17] M. Klessinger and J. Michl, Excited States and Photochemistry of Organic Molecules, VCH, New York, 1995.

[18] L. Ciano, P. Foggi, and P. R. Salvi, J. Photochem. Photobio. A 105 (1997), 129.

[19] L. Bussotti, P. Foggi, C. Gellini, L. Moroni, and P. R. Salvi, work to be published.

[20] F. V. R. Neuwhal and P. Foggi, Laser Chem. 19 (1999), 375.

[21] S. J. Formosinho and L. G. Arnaut, J. Photochem. Photobiol. A: Chem. 75 (1993), 21. 


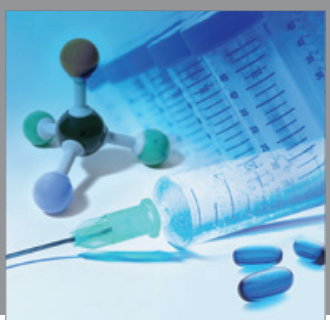

International Journal of

Medicinal Chemistry

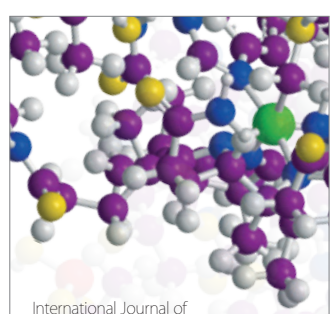

Carbohydrate Chemistry

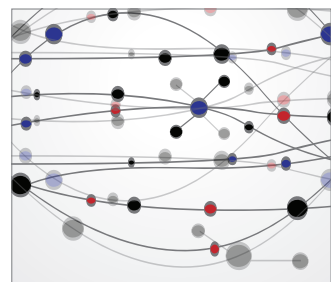

The Scientific World Journal
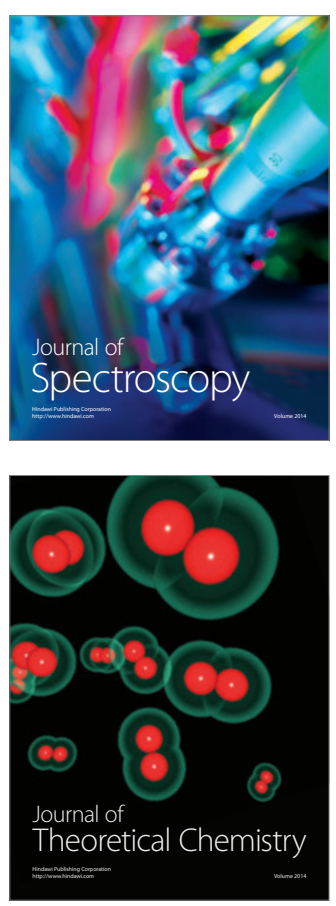
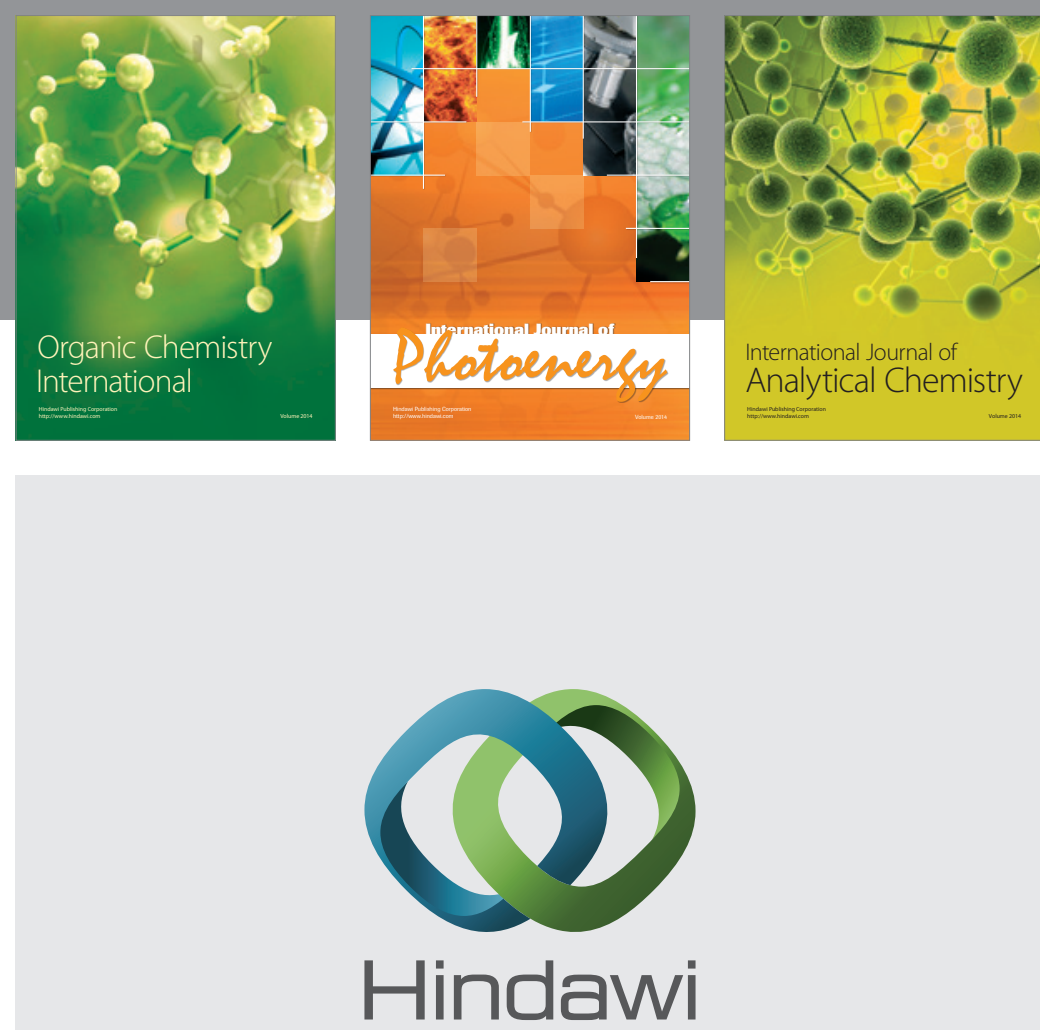

Submit your manuscripts at

http://www.hindawi.com
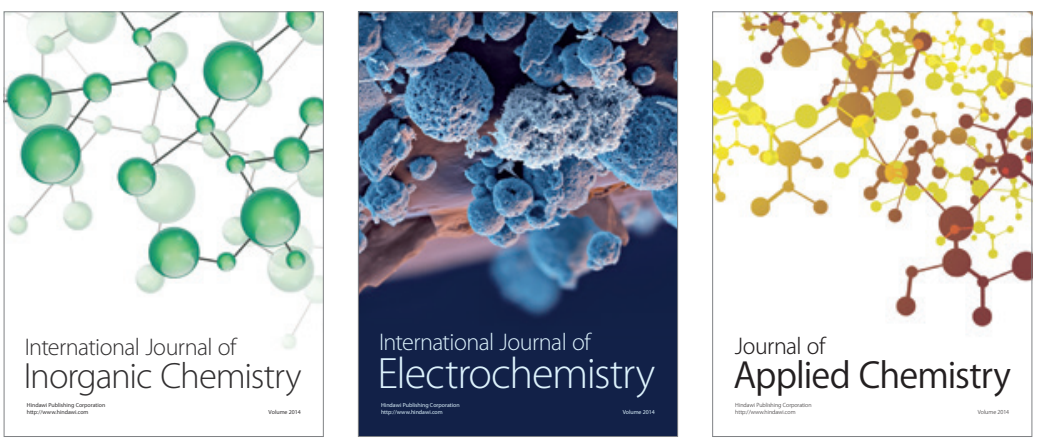

Journal of

Applied Chemistry
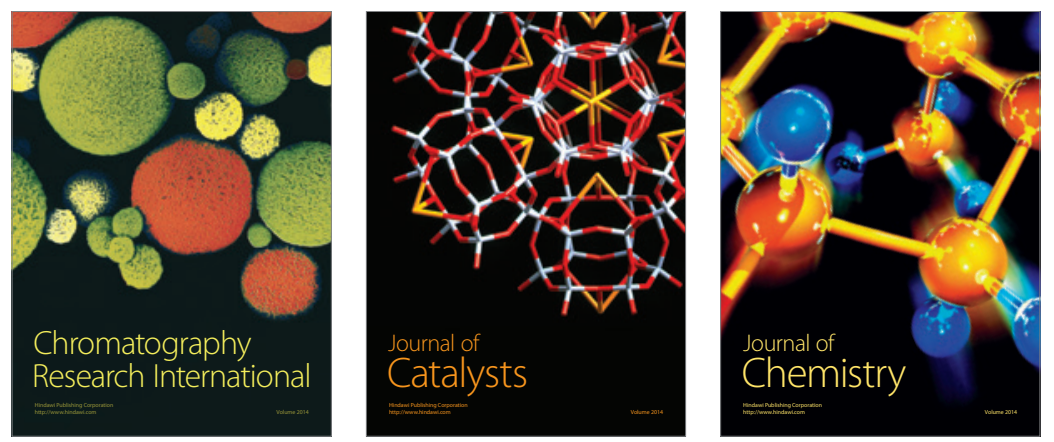
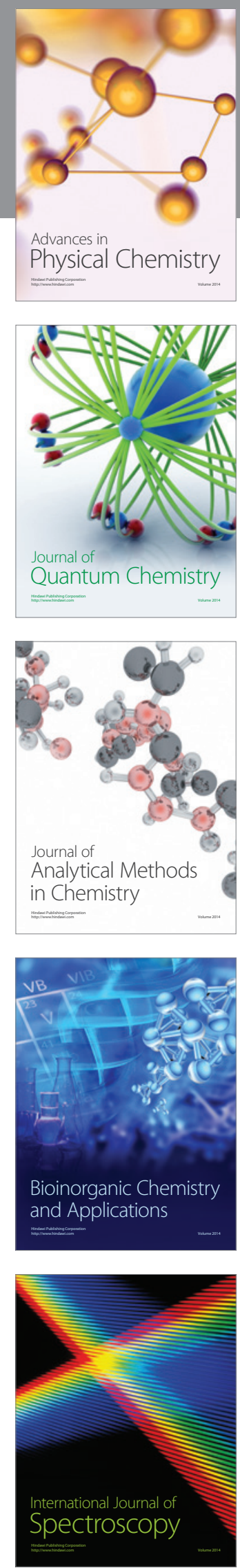